\title{
Application of a strong FSI coupling scheme for the numerical simulation of bileaflet mechanical heart valve dynamics: Study of wall shear stress on the valve leaflets
}

\section{Sebastiaan Annerel* ${ }^{*}$, Joris Degroote, Jan Vierendeels}

Department of Flow, Heat and Combustion Mechanics,

Ghent University (UGent),

9000 Ghent, Belgium

Email: Sebastiaan.Annerel@UGent.be

Email: Joris.Degroote@UGent.be

Email: Jan.Vierendeels@UGent.be

* Corresponding author

\section{Tom Claessens, Peter Van Ransbeeck}

Department of Mechanics,

University College Ghent,

9000 Ghent, Belgium

Email: Tom.Claessens@HoGent.be

Email: Peter.VanRansbeeck@hogent.be

\section{Sigrid K. Dahl, Bjørn Skallerud, Leif Rune Hellevik}

Department of Structural Engineering,

Norwegian University of Science and Technology (NTNU),

7491 Trondheim, Norway

Email: Sigrid.Dahl@ntnu.no

Email: Bjorn.Skallerud@ntnu.no

Email: Leif.R.Hellevik@ntnu.no

\section{Patrick Segers, Pascal Verdonck}

IBiTech-bioMMeda,

Ghent University (UGent),

9000 Ghent, Belgium

Email: Patrick.Segers@UGent.be

Email: Pascal.Verdonck@UGent.be

\begin{abstract}
One of the major challenges in the design of bileaflet mechanical heart valves (BMHVs) is reduction of the blood damage generated by non-physiological blood flow. Numerical simulations provide relevant insights into the (fluid) dynamics of the BMHV and are used for design optimization. In this paper, a strong coupling algorithm for the partitioned fluid-structure interaction (FSI) simulation of a BMHV is presented. The convergence of the coupling iterations between the flow solver and the leaflet motion solver is accelerated by using a numerically calculated Jacobian with the derivatives of the pressure and viscous moments acting on the leaflets with respect to the leaflet accelerations. The developed algorithm is used to simulate the dynamics of a 3D BMHV in three different geometries, allowing an analysis of the solution process. Moreover, the leaflet kinematics and the general flow field are discussed, with special focus on the shear stresses on the valve leaflets.
\end{abstract}

Keywords: Fluid-Structure Interaction, Algorithm, BMHV, Biomedical applications.

Reference to this paper should be made as follows: Annerel, S., Degroote, J., Claessens, T., Dahl, S.K., Skallerud, B., Hellevik, L.R., Van Ransbeeck, P., Segers, P., Verdonck, P. and Vierendeels, J. (2011) 'Application of a strong FSI coupling scheme for the numerical simulation of bileaflet mechanical heart valve dynamics: Study of wall shear stress on the valve leaflets', Progress in Computational Fluid Dynamics, Vol. ?, No. ?, pp.?-?. 
Biographical notes: S. Annerel graduated as Master of Science in Mechanical Engineering (2008) and Master of Science in Business Economics (2010), both from Ghent University in Belgium. Since September 2008, he is a PhD student at the Department of Flow, Heat and Combustion Mechanics of Ghent University. His main field of research is the numerical simulation of fluidstructure interaction applied to bileaflet mechanical heart valves.

J. Degroote received his BSc (2004), MSc (2006) and PhD (2010) in Mechanical Engineering from Ghent University in Belgium. Since October 2010, he is a post-doctoral fellow of the Research Foundation - Flanders (FWO). He was visiting researcher during one year at the Massachusetts Institute of Technology (United States of America) and three months at the Technical University of Munich (Germany). His research is devoted to multi-phase flow, high-performance computing, surrogate-based and gradient-based optimization and also the development and analysis of coupling algorithms for partitioned simulation of fluid-structure interaction.

J. Vierendeels was born in Dendermonde, Belgium, on November 27, 1968. He received the M.Sc. degree in electromechanical engineering, the M.Sc. degree in aeronautical and astronautical engineering, the M.Sc. degree in biomedical engineering, and the Ph.D. degree in electromechanical engineering from Ghent University, Ghent, Belgium, in 1991, 1993, 1996, and 1998, respectively. Currently, he is a full-time Professor with the Department of Flow, Heat, and Combustion Mechanics, Ghent University, working in the field of computational fluid dynamics and fluid-structure interaction.

T. Claessens has a background in civil and biomedical engineering, and received his $\mathrm{PhD}$ in Engineering from Ghent University in Belgium in 2006. Since 2007, he is working at the University College Ghent at the Faculty of Applied Engineering Sciences, where he teaches theoretical mechanics, fluid mechanics and thermodynamics. His fields of research are cardiovascular engineering (in particular cardiac biomechanics) and optical flow measurements techniques such as Particle Image Velocimetry.

$\mathrm{P}$. Van Ransbeeck has a background in aerospace engineering (TUDelft), and received his $\mathrm{PhD}$ in Engineering from University of Brussels in Belgium in 1998. He did a post-doc at the Von Karman Institute for Fluid Dynamics, and worked at the spin-off companies LMS International and ElSyCa, on respectively aero-acoustic and electrochemical numerical modeling. Since 2003, he is working at the University College Ghent at the Faculty of Applied Engineering Sciences, Department of Mechanics, where he teaches applied fluid mechanics and thermodynamics, engineering fluid dynamics and climate control. He is the head of a Mechanical Engineering Center where his expertise lies in CAE concurrent engineering using virtual and physical flow modeling technology.

P. Segers was born in Ninove, Belgium, on May 14, 1968. He received the degree of Civil Engineer in 1991, and the Ph.D. degree in applied sciences in 1997, both from Ghent University, Gent, Belgium. Since 2003, he has been a full-time research professor at the Institute Biomedical Technology, Ghent University. His current research interests include early noninvasive diagnosis of cardiovascular disease, with emphasis on large artery function and stiffness, and this through experimental and mathematical modeling and simulation, as well as in vivo research.

P. Verdonck was born in 1963 in Gent, Belgium. He graduated as Master of Science Civil Engineering in 1986 from the Ghent University. He obtained his diplomas in Master of Business Administration (1989), Master of Science Biomedical Engineering (1993) and PhD (1993) from the Ghent University. His research has spanned numerous fields of biomedical engineering sciences: experimental and numerical modeling of the cardiovascular system, transport phenomena in extracorporeal circulation and artificial organs and computational biodesign.

S.K. Dahl received her MSc (2006) in Mechanical Engineering from the Norwegian University of Science and Technology (NTNU), Trondheim, Norway. She is now a doctorial candidate at NTNU. Her research is mainly focusing on numerical simulations of blood flow in the left side of the heart.

B. Skallerud is full professor in Computational Mechanics since 1997, and head of Biomechanics Division since 2005, both positions at NTNU, Department of Structural Engineering, Trondheim, Norway. He received his PhD in Structural Mechanics in 1988. From 1988 to 1997 he worked with contract research related to offshore structures at SINTEF, Trondheim, Norway.

L.R. Hellevik (born 1967) is a full professor in Biomechanics since 2006 at NTNU, Trondheim, Norway. He received his MSc in applied mathematics in 1991, and his $\mathrm{PhD}$ in biomechanics in 1999. From 1999 to 2006 he worked with contract research related to flow technology at SINTEF, Trondheim, Norway. 


\section{$1 \quad$ Introduction}

Since the first clinical success with an artificial heart valve by Dr. Hufnagel in 1952, the implantation of valve prostheses has become a routine treatment for severe heart valve failure. However, despite sixty years of research, modern valve prostheses still have severe design deficiencies. The widely used and preferred bileaflet mechanical heart valves (BMHVs), for example, still have poor hemodynamic properties and patients need to undergo life-long anti-coagulation therapies (Dasi et al. 2009). Therefore, one of the major challenges in the design of BMHVs is the reduction of the accumulated blood damage which is, among others, caused by non-physiological flow patterns and elevated shear stress levels on the leaflets. Numerical simulations of the (blood) flow provide relevant insights into the valve dynamics (Dasi et al. 2009) and can be used for design optimization. Unfortunately, such numerical simulation of a BMHV is a complex fluidstructure interaction (FSI) problem because the movement of the leaflets strongly interacts with the surrounding fluid motion and, therefore, the equilibrium at the fluid-structure interface needs to be taken into account.

In this paper, a strong coupling algorithm for the simulation of a BMHV by a partitioned solver is presented and used to study the wall shear stresses on the valve leaflet surfaces. The algorithm predicts the leaflet moments (and thus the leaflet angular accelerations) of the next coupling iteration through a linearization of Newton's Second Law with a finite difference approximation of the Jacobian. The components of this Jacobian are the derivatives of the moments (exerted by the flow on the leaflets) with respect to the leaflet angular accelerations. The Jacobian is numerically calculated from the flow solver by variations of the leaflet positions. This quasi-Newton method was first introduced in Vierendeels et al. (2005) for one stiff leaflet and subsequently used to simulate a BMHV (Dumont et al. 2005, 2007). However, the algorithm described in Vierendeels et al. (2005) had only one degree of freedom which implied a symmetrical motion of both leaflets when simulating a BMHV. Therefore, the algorithm was extended to two degrees of freedom in Annerel et al. (2010) and Dahl et al. (2010), thus allowing the two leaflets to move asynchronously. In this paper, the convergence process is accelerated by the use of a variable time step size, extrapolation techniques and reuse of data from previous time steps. Also, the calculation process of the Jacobian is made more efficient, thus resulting in faster convergence.

The paper is organized as follows. First, the algorithm is derived and its implementation in a commercially available black box flow solver is analysed. Secondly, the algorithm is used to simulate a $3 \mathrm{D}$ BMHV in three different geometries, allowing an analysis of the leaflets kinematics and the wall shear stresses on the valve leaflet surfaces.

\section{$2 \quad$ Methods}

In this section, the developed algorithm is presented. The flow chart is visualized in Figure 2 and each component is explained hereafter. Subsequently, the setup of the 3D simulations of the BMHV is discussed.

\subsection{FSI coupling algorithm}

\subsubsection{Governing equations}

A BMHV can be modelled as a rigid casing in which two separate rigid leaflets rotate around their hinge axes (see Figure 1). Because the position of each rigid leaflet is solely determined by its opening angle, the bileaflet valve has two degrees of freedom.

The movement of a rigid leaflet $i$ is governed by Newton's Second Law, which states that the moment about its hinge $\left(M_{i}\right)$ must be in equilibrium with the product of its moment of inertia $\left(I_{i}\right)$ and its angular acceleration $\left(\ddot{\theta}_{i}\right)$. For two leaflets, this leads to the following two equations:

$$
\left\{\begin{array}{l}
M_{1}=I_{1} \cdot \ddot{\theta}_{1} \\
M_{2}=I_{2} \cdot \ddot{\theta}_{2}
\end{array}\right.
$$

When the hinges are modelled as frictionless, the moment acting on the leaflet is the pressure and viscous moment exerted by the flow.

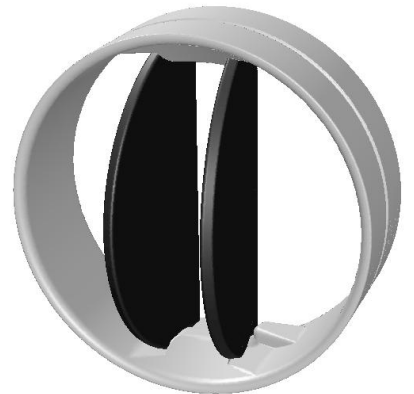

Figure 1: View on the ATS Open Pivot ${ }^{\mathrm{TM}}$ Standard Heart Valve with leaflets (marked in black) in the open position. The casing is visible (in white) with the blocking mechanism at the hinges.

\subsubsection{Linearization}

Strong coupling schemes achieve this dynamic equilibrium at the fluid-structure interface by introducing a coupling iteration loop within each time step. Generally, each of the coupling iterations follows the same pattern, as is visualized in Figure 2 (shaded). At the beginning of each coupling iteration $k$ of time step $n+1$, the motion of the leaflets is computed from the angular accelerations $\ddot{\theta}_{i}^{n+1, k}$. Subsequently, the mesh is moved and the Navier-Stokes equations are solved. From the flow field, the moments $M_{i}^{n+1, k}$ are calculated. Finally, the convergence of the dynamic equilibrium at the fluid-structure interface (i.e. the "FSI convergence", expressed by Equation (1)) is checked. When this FSI convergence is obtained, a new time step is initiated. However, when the FSI convergence is not achieved, a new coupling iteration $k+1$ is initiated and thus new angular accelerations $\ddot{\theta}_{i}^{n+1, k+1}$ need to be calculated. Therefore, the introduction of a coupling iteration loop requires, in each coupling iteration $k$ of time step $n+1$, a stable and efficient approximation of the angular accelerations for the next coupling iteration $k+1$.

Such a stable prediction of $\ddot{\theta}_{i}^{n+1, k}$ can be achieved through a linearization of Newton's Second Law, as analysed by Vierendeels et al. (2005) for a monoleaflet heart valve. Thus, while taking into account the mutual interaction 


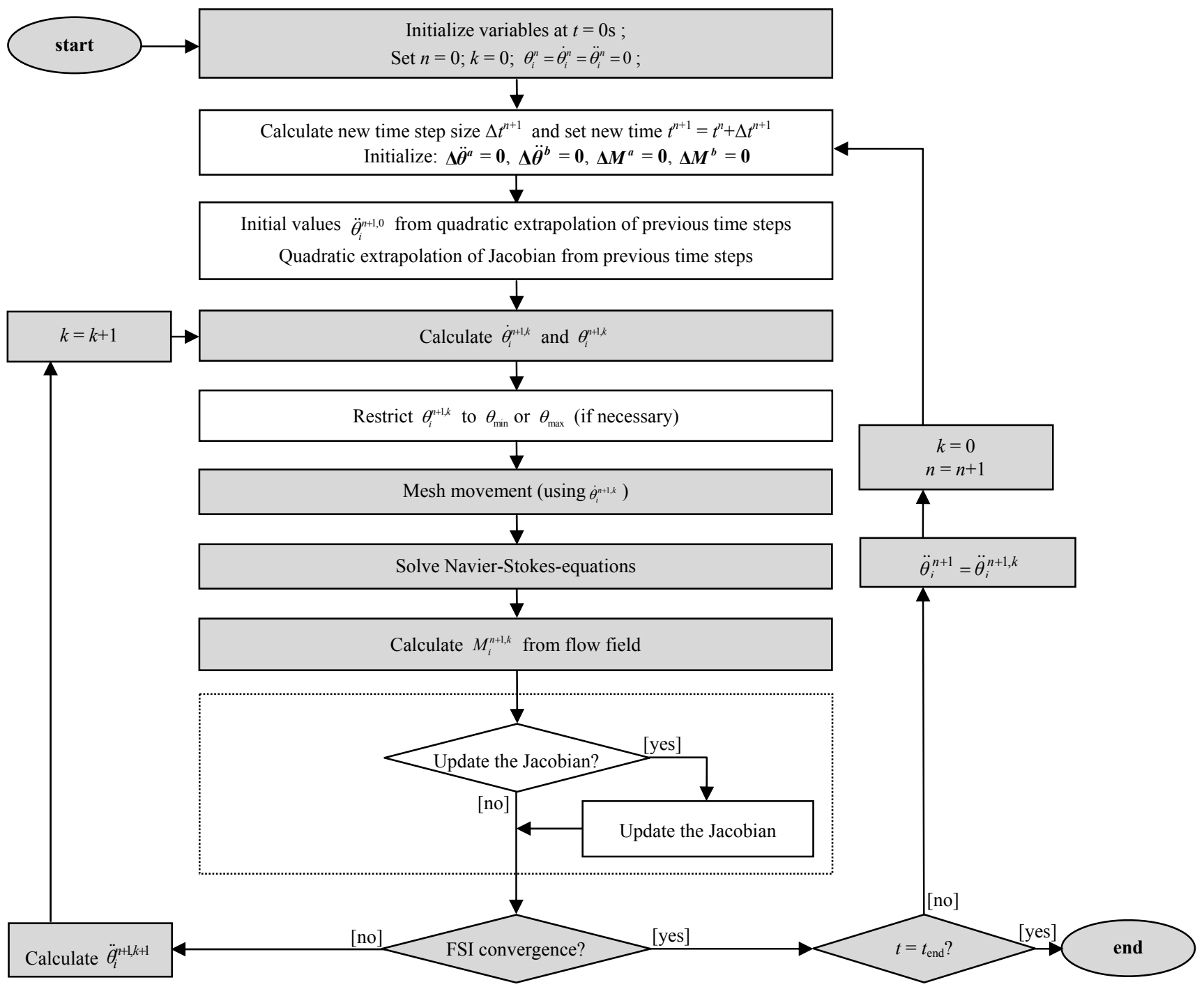

Figure 2: Flow chart of the presented quasi-Newton coupling algorithm with two degrees of freedom. $n=$ time step, $k=$ coupling iteration step, $i=$ leaflet number. Shaded: standard strong coupling scheme, unshaded: extra components of the presented scheme.

between the leaflets, Equation (1) is linearized for each coupling iteration $k+1$ of time step $n+1$ :

$$
\left\{\begin{array}{l}
M_{1}^{n+1, k}+\left(\frac{\partial M_{1}}{\partial \ddot{\theta}_{1}}\right)^{n+1, k}\left(\ddot{\theta}_{1}^{n+1, k+1}-\ddot{\theta}_{1}^{n+1, k}\right)+\left(\frac{\partial M_{1}}{\partial \ddot{\theta}_{2}}\right)^{n+1, k}\left(\ddot{\theta}_{2}^{n+1, k+1}-\ddot{\theta}_{2}^{n+1, k}\right)=I_{1} \cdot \ddot{\theta}_{1}^{n+1, k+1} \\
M_{2}^{n+1, k}+\left(\frac{\partial M_{2}}{\partial \ddot{\theta}_{1}}\right)^{n+1, k}\left(\ddot{\theta}_{1}^{n+1, k+1}-\ddot{\theta}_{1}^{n+1, k}\right)+\left(\frac{\partial M_{2}}{\partial \ddot{\theta}_{2}}\right)^{n+1, k}\left(\ddot{\theta}_{2}^{n+1, k+1}-\ddot{\theta}_{2}^{n+1, k}\right)=I_{2} \cdot \ddot{\theta}_{2}^{n+1, k+1}
\end{array}\right.
$$

The derivatives $\partial M_{i} / \partial \ddot{\theta}_{j}$ are the components of the Jacobian of the moments with respect to the angular accelerations, further referred to as "the Jacobian". Equation (2) can be rearranged as follows:

$$
\left\{\begin{array}{l}
I_{1} \cdot \ddot{\theta}_{1}^{n+1, k+1}-\left(\frac{\partial M_{1}}{\partial \ddot{\theta}_{1}}\right)^{n+1, k} \ddot{\theta}_{1}^{n+1, k+1}-\left(\frac{\partial M_{1}}{\partial \ddot{\theta}_{2}}\right)^{n+1, k} \ddot{\theta}_{2}^{n+1, k+1}=M_{1}^{n+1, k}-\left(\frac{\partial M_{1}}{\partial \ddot{\theta}_{1}}\right)^{n+1, k} \ddot{\theta}_{1}^{n+1, k}-\left(\frac{\partial M_{1}}{\partial \ddot{\theta}_{2}}\right)^{n+1, k} \ddot{\theta}_{2}^{n+1, k} \\
I_{2} \cdot \ddot{\theta}_{2}^{n+1, k+1}-\left(\frac{\partial M_{2}}{\partial \ddot{\theta}_{1}}\right)^{n+1, k} \ddot{\theta}_{1}^{n+1, k+1}-\left(\frac{\partial M_{2}}{\partial \ddot{\theta}_{2}}\right)^{n+1, k} \ddot{\theta}_{2}^{n+1, k+1}=M_{2}^{n+1, k}-\left(\frac{\partial M_{2}}{\partial \ddot{\theta}_{1}}\right)^{n+1, k} \ddot{\theta}_{1}^{n+1, k}-\left(\frac{\partial M_{2}}{\partial \ddot{\theta}_{2}}\right)^{n+1, k} \ddot{\theta}_{2}^{n+1, k}
\end{array}\right.
$$

This can be written in matrix notation:

$$
\left[\begin{array}{cc}
I_{1}-\left(\frac{\partial M_{1}}{\partial \ddot{\theta}_{1}}\right)^{n+1, k} & -\left(\frac{\partial M_{1}}{\partial \ddot{\theta}_{2}}\right)^{n+1, k} \\
-\left(\frac{\partial M_{2}}{\partial \ddot{\theta}_{1}}\right)^{n+1, k} & I_{2}-\left(\frac{\partial M_{2}}{\partial \ddot{\theta}_{2}}\right)^{n+1, k}
\end{array}\right] \cdot\left[\begin{array}{l}
\ddot{\theta}_{1}^{n+1, k+1} \\
\ddot{\theta}_{2}^{n+1, k+1}
\end{array}\right]=\left[\begin{array}{l}
M_{1}^{n+1, k}-\left(\frac{\partial M_{1}}{\partial \ddot{\theta}_{1}}\right)^{n+1, k} \ddot{\theta}_{1}^{n+1, k}-\left(\frac{\partial M_{1}}{\partial \ddot{\theta}_{2}}\right)^{n+1, k} \ddot{\theta}_{2}^{n+1, k} \\
M_{2}^{n+1, k}-\left(\frac{\partial M_{2}}{\partial \ddot{\theta}_{1}}\right)^{n+1, k} \ddot{\theta}_{1}^{n+1, k}-\left(\frac{\partial M_{2}}{\partial \ddot{\theta}_{2}}\right)^{n+1, k} \ddot{\theta}_{2}^{n+1, k}
\end{array}\right]
$$

For a well-posed problem, the first matrix has to be nonsingular. In that case, previous equation can be rewritten as

$$
\left[\begin{array}{l}
\ddot{\theta}_{1}^{n+1, k+1} \\
\ddot{\theta}_{2}^{n+1, k+1}
\end{array}\right]=\left[\begin{array}{cc}
I_{1}-\left(\frac{\partial M_{1}}{\partial \ddot{\theta}_{1}}\right)^{n+1, k} & -\left(\frac{\partial M_{1}}{\partial \ddot{\theta}_{2}}\right)^{n+1, k} \\
-\left(\frac{\partial M_{2}}{\partial \ddot{\theta}_{1}}\right)^{n+1, k} & I_{2}-\left(\frac{\partial M_{2}}{\partial \ddot{\theta}_{2}}\right)^{n+1, k}
\end{array}\right]^{-1}\left[\begin{array}{l}
M_{1}^{n+1, k}-\left(\frac{\partial M_{1}}{\partial \ddot{\theta}_{1}}\right)^{n+1, k} \ddot{\theta}_{1}^{n+1, k}-\left(\frac{\partial M_{1}}{\partial \ddot{\theta}_{2}}\right)^{n+1, k} \ddot{\theta}_{2}^{n+1, k} \\
M_{2}^{n+1, k}-\left(\frac{\partial M_{2}}{\partial \ddot{\theta}_{1}}\right)^{n+1, k} \ddot{\theta}_{1}^{n+1, k}-\left(\frac{\partial M_{2}}{\partial \ddot{\theta}_{2}}\right)^{n+1, k} \ddot{\theta}_{2}^{n+1, k}
\end{array}\right]
$$

In order to solve Equation (5) and thus to calculate the angular accelerations of the new coupling iteration $k+1$, the Jacobian has to be known. However, when a black box flow solver is used, these derivatives are not accessible. Fortunately, they can be approximated by finite differences $(i, j=1,2)$ :

$$
\frac{\partial M_{i}}{\partial \ddot{\theta}_{j}} \approx \frac{\Delta M_{i}}{\Delta \ddot{\theta}_{j}}
$$

which are calculated by solving the following system of equations: 


$$
\left\{\begin{array}{l}
\left(\frac{\Delta M_{1}}{\Delta \ddot{\theta}_{1}}\right) \cdot \Delta \ddot{\theta}_{1}^{a}+\left(\frac{\Delta M_{1}}{\Delta \ddot{\theta}_{2}}\right) \cdot \Delta \ddot{\theta}_{2}^{a}=\Delta M_{1}^{a} \\
\left(\frac{\Delta M_{2}}{\Delta \ddot{\theta}_{1}}\right) \cdot \Delta \ddot{\theta}_{1}^{a}+\left(\frac{\Delta M_{2}}{\Delta \ddot{\theta}_{2}}\right) \cdot \Delta \ddot{\theta}_{2}^{a}=\Delta M_{2}^{a} \\
\left(\frac{\Delta M_{1}}{\Delta \ddot{\theta}_{1}}\right) \cdot \Delta \ddot{\theta}_{1}^{b}+\left(\frac{\Delta M_{1}}{\Delta \ddot{\theta}_{2}}\right) \cdot \Delta \ddot{\theta}_{2}^{b}=\Delta M_{1}^{b} \\
\left(\frac{\Delta M_{2}}{\Delta \ddot{\theta}_{1}}\right) \cdot \Delta \ddot{\theta}_{1}^{b}+\left(\frac{\Delta M_{2}}{\Delta \ddot{\theta}_{2}}\right) \cdot \Delta \ddot{\theta}_{2}^{b}=\Delta M_{2}^{b}
\end{array}\right.
$$

resulting in

$$
\left\{\begin{array}{l}
\left(\frac{\Delta M_{1}}{\Delta \ddot{\theta}_{1}}\right)=\frac{\Delta M_{1}^{a} \cdot \Delta \ddot{\theta}_{2}^{b}-\Delta M_{1}^{b} \cdot \Delta \ddot{\theta}_{2}^{a}}{\Delta \ddot{\theta}_{1}^{a} \cdot \Delta \ddot{\theta}_{2}^{b}-\Delta \ddot{\theta}_{1}^{b} \cdot \Delta \ddot{\theta}_{2}^{a}} \\
\left(\frac{\Delta M_{1}}{\Delta \ddot{\theta}_{2}}\right)=\frac{\Delta M_{1}^{b} \cdot \Delta \ddot{\theta}_{1}^{a}-\Delta M_{1}^{a} \cdot \Delta \ddot{\theta}_{1}^{b}}{\Delta \ddot{\theta}_{1}^{a} \cdot \Delta \ddot{\theta}_{2}^{b}-\Delta \ddot{\theta}_{1}^{b} \cdot \Delta \ddot{\theta}_{2}^{a}} \\
\left(\frac{\Delta M_{2}}{\Delta \ddot{\theta}_{1}}\right)=\frac{\Delta M_{2}^{a} \cdot \Delta \ddot{\theta}_{2}^{b}-\Delta M_{2}^{b} \cdot \Delta \ddot{\theta}_{2}^{a}}{\Delta \ddot{\theta}_{1}^{a} \cdot \Delta \ddot{\theta}_{2}^{b}-\Delta \ddot{\theta}_{1}^{b} \cdot \Delta \ddot{\theta}_{2}^{a}} \\
\left(\frac{\Delta M_{2}}{\Delta \ddot{\theta}_{2}}\right)=\frac{\Delta M_{2}^{b} \cdot \Delta \ddot{\theta}_{1}^{a}-\Delta M_{2}^{a} \cdot \Delta \ddot{\theta}_{1}^{b}}{\Delta \ddot{\theta}_{1}^{a} \cdot \Delta \ddot{\theta}_{2}^{b}-\Delta \ddot{\theta}_{1}^{b} \cdot \Delta \ddot{\theta}_{2}^{a}}
\end{array}\right.
$$

The superscripts $a$ and $b$ refer to data obtained from three coupling iterations between which the leaflet angular accelerations of two coupling iterations have differences (according to the vectors $\Delta \ddot{\theta}^{a}$ and $\Delta \ddot{\theta}^{b}$ ) with respect to the angular accelerations of a reference coupling iteration:

$$
\begin{aligned}
\boldsymbol{\Delta} \ddot{\boldsymbol{\theta}}^{a} & =\left[\begin{array}{ll}
\ddot{\theta}_{1}^{a}-\ddot{\theta}_{1}^{r e f} \\
\ddot{\theta}_{2}^{a}-\ddot{\theta}_{2}^{r e f}
\end{array}\right]=\left[\begin{array}{c}
\ddot{\theta}_{1}^{a} \\
\ddot{\theta}_{2}^{a}
\end{array}\right] \\
\boldsymbol{\Delta} \ddot{\boldsymbol{\theta}}^{b} & =\left[\begin{array}{l}
\ddot{\theta}_{1}^{b}-\ddot{\theta}_{1}^{r e f} \\
\ddot{\theta}_{2}^{b}-\ddot{\theta}_{2}^{r e f}
\end{array}\right]=\left[\begin{array}{c}
\Delta \ddot{\theta}_{1}^{b} \\
\Delta \ddot{\theta}_{2}^{b}
\end{array}\right]
\end{aligned}
$$

These differences in accelerations $\left(\Delta \ddot{\theta}^{a}\right.$ and $\left.\Delta \ddot{\theta}^{b}\right)$ induce differences in the calculated flow fields and thus also in the calculated moments, respectively denoted by $\Delta \boldsymbol{M}^{a}$ and $\boldsymbol{\Delta} \boldsymbol{M}^{b}$ :

$$
\begin{aligned}
& \Delta \boldsymbol{M}^{\boldsymbol{a}}=\left[\begin{array}{l}
M_{1}^{a}-M_{1}^{r e f} \\
M_{2}^{a}-M_{2}^{r e f}
\end{array}\right]=\left[\begin{array}{l}
\Delta M_{1}^{a} \\
\Delta M_{2}^{a}
\end{array}\right] \\
& \boldsymbol{\Delta M}^{\boldsymbol{b}}=\left[\begin{array}{l}
M_{1}^{b}-M_{1}^{r e f} \\
M_{2}^{b}-M_{2}^{r e f}
\end{array}\right]=\left[\begin{array}{l}
\Delta M_{1}^{b} \\
\Delta M_{2}^{b}
\end{array}\right]
\end{aligned}
$$

The data $\Delta \ddot{\theta}^{a}, \Delta \ddot{\theta}^{b}, \Delta \boldsymbol{M}^{a}$ and $\Delta \boldsymbol{M}^{b}$ can be obtained from three coupling iterations by several methods.

\subsubsection{Update of the Jacobian}

A method to obtain the necessary data $\left(\Delta \ddot{\theta}^{a}, \Delta \ddot{\theta}^{b}, \Delta \boldsymbol{M}^{a}\right.$ and $\Delta \boldsymbol{M}^{b}$ ) is described in Annerel et al. (2010) and Dahl et al. (2010). In this method, three consecutive coupling iterations are needed of which the leaflet acceleration perturbation vectors $\Delta \ddot{\theta}^{a}$ and $\Delta \ddot{\theta}^{b}$ are perpendicular to each other and significantly large.

This method can be improved in two ways. First, it is not necessary that the acceleration perturbation vectors $\Delta \ddot{\theta}^{a}$ and $\Delta \ddot{\theta}^{b}$ are completely perpendicular to each other. To calculate a good estimation of the Jacobian, it is already sufficient that their perpendicular components are significantly large. Secondly, the three coupling iterations do not have to be consecutive.

The calculation of the Jacobian can thus be made more efficient by the use of a criterion that selects two appropriate acceleration perturbation vectors (e.g. from previous coupling iterations), without the need for extra coupling iterations with perpendicularly perturbed accelerations. Therefore, the selection criterion needs to check the direction and the magnitude of the two acceleration perturbation vectors. Such a criterion can be derived from an error propagation study, which states that the error of the estimation of the angular acceleration of the next coupling iteration should be small enough. It can be shown that this results in the following criterion (Annerel et al. 2011):

$$
\frac{\max _{i=1,2}\left(\sqrt{\left(\Delta \ddot{\theta}_{i}^{a}\right)^{2}+\left(\Delta \ddot{\theta}_{i}^{b}\right)^{2}}\right)}{\left|\Delta \ddot{\theta}_{2}^{b} \cdot \Delta \ddot{\theta}_{1}^{a}-\Delta \ddot{\theta}_{2}^{a} \cdot \Delta \ddot{\theta}_{1}^{b}\right|} \cdot \frac{\varepsilon_{1}}{\min _{i=1,2}\left(I_{i}\right)} \leq \varepsilon_{2}
$$

with the Jacobian update threshold $\varepsilon_{2}$ and the flow solver convergence threshold $\varepsilon_{1}$, which is the accuracy of the moments calculated by the flow solver. The optimal values for $\varepsilon_{1}$ and $\varepsilon_{2}$ can be determined by a sensitivity analysis.

This criterion is used in an algorithm that makes it possible to update the Jacobian in every coupling iteration with data obtained from the coupling iterations in the current and previous time steps. This algorithm for the update of the Jacobian is constructed as follows:

At the beginning of the simulation, all the variables are equal to zero (in particular $\Delta \ddot{\theta}^{a}, \Delta \ddot{\theta}^{b}, \Delta \boldsymbol{M}^{a}, \Delta \boldsymbol{M}^{b}$ and all the components of the Jacobian). Also, when going to each new time step, the data vectors $\left(\Delta \ddot{\theta}^{a}, \Delta \ddot{\theta}^{b}, \Delta \boldsymbol{M}^{a}\right.$ and $\left.\Delta \boldsymbol{M}^{b}\right)$ are set to zero (see further).

The data obtained in the first coupling iteration $(k=0)$ of each time step are used as the reference (i.e. $\ddot{\theta}_{i}^{\text {ref }}$ and $M_{i}^{\text {ref }}$ ) for the Jacobian update. Also, in this first coupling iteration a first estimation of the Jacobian is obtained from an extrapolation of previous time steps (see further).

In each of the following coupling iterations $(k>0)$, this Jacobian can be updated with useable data obtained in the coupling iterations. First, it is checked whether or not the data of the current coupling iteration $k$ are useable as an acceleration perturbation vector. Subsequently, the Jacobian can be updated in three ways, depending on the acceleration perturbation vectors $\Delta \ddot{\theta}^{a}$ and $\Delta \ddot{\theta}^{b}$ available from the (current and previous) coupling iterations (a detailed description can be found in Annerel et al. (2011)):

- If no appropriate acceleration perturbation vectors are available, then the Jacobian cannot be updated and thus the extrapolated Jacobian is still used;

- If one acceleration perturbation vector is available, then the Jacobian can be updated. The data for the other acceleration perturbation vector are obtained from the acceleration perturbation vectors of previous time steps, or this data can be calculated by the extrapolated Jacobian;

- If both acceleration perturbation vectors are available, the Jacobian is calculated with both vectors and becomes independent of data from previous time steps.

In the following, the other components of the developed quasi-Newton algorithm are discussed. The flow chart of this algorithm is visualized in Figure 2.

\subsubsection{Check FSI convergence}

The subsequent coupling iterations are performed until the dynamic equilibrium at the fluid-structure interface is achieved. This equilibrium is checked by the FSI 
convergence criterion. For each leaflet $i$, this FSI convergence criterion is given by:

$$
\left|M_{i}^{n+1, k}-I_{i} \cdot \ddot{\theta}_{i}^{n+1, k}\right|<\varepsilon_{0}
$$

The FSI convergence threshold $\varepsilon_{0}$ is set relative to a reference moment $M_{\text {ref }}$, which sets the minimal accuracy of the dynamic equilibrium needed to capture the general leaflet dynamics.

When the FSI convergence criterion (Equation (12)) is satisfied, a new time step is started. However, when the FSI convergence criterion is not satisfied, then a new coupling iteration $(k+1)$ is initiated.

\subsubsection{Control quality of the Jacobian and calculate angular accelerations of the new coupling iteration}

Before calculating the appropriate angular accelerations $\ddot{\theta}_{i}^{n+1, k+1}$ for the new coupling iteration $k+1$, the quality of the Jacobian is checked by evaluating the decrease in moment unbalance during the last two coupling iterations. For example, an arbitrary criterion of two orders of magnitude between these two consecutive coupling iterations is used:

$$
\left|M_{i}^{n+1, k}-I_{i} \cdot \ddot{\theta}_{i}^{n+1, k}\right|<\frac{\left|M_{i}^{n+1, k-1}-I_{i} \cdot \ddot{\theta}_{i}^{n+1, k-1}\right|}{100}
$$

When this expression is fulfilled, then the quality of the Jacobian of coupling iteration $k-1$ is good. Therefore, it can be concluded that the Jacobian of the current coupling iteration $k$ will probably also result in fast convergence, since this Jacobian can only become an improvement of the Jacobian of coupling iteration $k-1$. This is because when the Jacobian is updated in coupling iteration $k$, this update is done with more (or equally) relevant data (i.e. data of time step $n+1$ and not of time step $n$ ) than was available in coupling iteration $k-1$. Subsequently, the angular accelerations of the next coupling iteration $k+1$ are calculated by Equation (5) with the Jacobian of coupling iteration $k$ and this next coupling iteration is initiated.

However, when Equation (13) is not fulfilled, then the quality of the Jacobian of coupling iteration $k-1$ is insufficient. Therefore, it is uncertain whether or not the Jacobian of coupling iteration $k$ will result in fast convergence. The angular accelerations of the next coupling iteration $\ddot{\theta}_{i}^{n+1, k+1}$ are thus chosen in such a way that it will certainly become possible to calculate a good Jacobian in the next coupling iteration using the acceleration and moment data generated in this next coupling iteration.

The angular acceleration of this extra coupling iteration is thus chosen in one of three possible ways, depending on the acceleration perturbation vectors $\Delta \ddot{\theta}^{a}$ and $\Delta \ddot{\theta}^{b}$ already obtained in the coupling iterations (a detailed description can be found in Annerel et al. (2011)):

- If no acceleration perturbation vectors are available from the coupling iterations (i.e $\Delta \ddot{\theta}^{a}=0$ and $\Delta \ddot{\theta}^{b}=0$ ), then the angular acceleration of one leaflet is perturbed with a significant parameter $\delta$ in the next coupling iteration, as is described in (Annerel et al. 2010, Dahl et al. 2010);

- If one acceleration perturbation vector is already available (i.e. $\Delta \ddot{\boldsymbol{\theta}}^{a} \neq 0$ and $\Delta \ddot{\boldsymbol{\theta}}^{b}=0$ ), then the leaflet accelerations of the next coupling iteration are perturbed in a direction that is perpendicular to $\Delta \ddot{\boldsymbol{\theta}}^{a}$;
- If both acceleration perturbation vectors are available (i.e $\Delta \ddot{\theta}^{a} \neq 0$ and $\Delta \ddot{\theta}^{b} \neq 0$ ), then no extra coupling iteration is needed. Therefore, the Jacobian is kept since the slow residual drop rate is inherent to the problem and will mostly not be efficiently resolved by generating extra data. The angular acceleration of the next coupling iterations are thus calculated by Equation (5).

\subsubsection{Increasing the efficiency}

The efficiency of the algorithm is increased in several ways. In the following, the use of a variable time step size and the extrapolation of data from previous time steps are discussed.

Variable time step size. Since a heart valve is most of the time in the closed or open position, its leaflets are only moving in a very small fraction of the time cycle. However, when the leaflets are moving, very small time steps are needed for reasons of accuracy. Therefore, there is a clear advantage in the use of a variable time step size which allows a relatively large time step size when the valve is at rest, and a smaller time step size when the valve is moving, since it decreases the total number of time steps in a time cycle and thus lowers the computational cost. For these reasons, a variable time step size is commonly used when simulating heart valves (Bang et al. 2006; Choi et al. 2009; De Tullio et al. 2009).

When the leaflets are moving, the maximum allowable time step size is restricted by the maximum allowable mesh motion, and thus by grid characteristics. This is due to limitations of the mesh motion algorithm, since the remeshing occurs when going to a new time step, and in the coupling iterations within a time step only grid node relocation can be used. So, there is a maximum allowable mesh motion in order to retain an accurate and high-quality mesh (and, in worst case, to avoid highly skewed or even inverted cells).

A criterion for the time step size can be proposed based on this maximum leaflet movement $\left(\Delta \theta_{\max }\right)$ :

$$
\dot{\theta}_{i}^{n} \cdot \Delta t^{n+1} \leq \Delta \theta_{\max } \Rightarrow \Delta t^{n+1} \leq \min _{i=1,2}\left(\frac{\Delta \theta_{\max }}{\dot{\theta}_{i}^{n}}\right)
$$

When the leaflet velocity is large, then the time step size will be small and vice versa. Furthermore, the time step size is limited by a maximal value $\Delta t^{\max }$, otherwise the time step size would become infinite when none of the leaflets is moving (i.e. zero velocity).

Extrapolation from previous time steps. When advancing to a new time step, the angular accelerations for the first coupling iteration $(k=0)$ are estimated based on a quadratic extrapolation from previous time steps. This initial prediction of the values results in fewer coupling iterations per time step.

Also, the Jacobian's components are quadratically extrapolated from previous time steps. This is meaningful since the Jacobian is not changing much per time step.

Therefore, the extrapolation of data from previous time steps decreases the number of coupling iterations per time step and lowers the computational cost. 


\subsubsection{Initialization at the beginning of a new time step}

When going to a new time step $n+1$, the variables are initialized. This is done by setting $t^{n+1}=t^{n}+\Delta t^{n+1}, \Delta \ddot{\boldsymbol{\theta}}^{a}=\mathbf{0}$, $\Delta \ddot{\theta}^{b}=\mathbf{0}, \Delta \boldsymbol{M}^{a}=\mathbf{0}, \Delta \boldsymbol{M}^{b}=\mathbf{0}$ and $k=0$.

\subsubsection{Time integration scheme and leaflet behaviour at the limited position}

Backward Euler time integration. The angular velocity and angular position of each leaflet on the time level $n+1$ are calculated from the angular accelerations using a timeintegration scheme. Because the flow solver in which the algorithm is implemented uses a backward Euler scheme, this scheme is also preferred for the structural movement:

$$
\left\{\begin{array} { l } 
{ \ddot { \theta } _ { i } ^ { n + 1 , k + 1 } = \frac { \dot { \theta } _ { i } ^ { n + 1 , k + 1 } - \dot { \theta } _ { i } ^ { n } } { \Delta t } } \\
{ \dot { \theta } _ { i } ^ { n + 1 , k + 1 } = \frac { \theta _ { i } ^ { n + 1 , k + 1 } - \theta _ { i } ^ { n } } { \Delta t } }
\end{array} \Rightarrow \left\{\begin{array}{l}
\dot{\theta}_{i}^{n+1, k+1}=\dot{\theta}_{i}^{n}+\Delta t \cdot \ddot{\theta}_{i}^{n+1, k+1} \\
\theta_{i}^{n+1, k+1}=\theta_{i}^{n}+\Delta t \cdot \dot{\theta}_{i}^{n}+\Delta t^{2} \cdot \ddot{\theta}_{i}^{n+1, k+1}
\end{array}\right.\right.
$$

If the time discretization schemes of the flow and the structural solver do not match, then spurious oscillations can occur, as is shown in Vierendeels et al. (2005).

Leaflet behaviour in open and closed position. The angular position of each leaflet is restricted by a minimum and a maximum angle, indicating, respectively, the fully closed and fully open position. In reality, this is usually achieved by a physical blocking mechanism incorporated in the design of the leaflet hinges. These limitations can be numerically modelled by setting the angular position equal to the limited position and the angular velocity and angular acceleration equal to zero, as briefly suggested by Guivier et al. (2007). However, they did not explain how to make the transition from a moving to a resting leaflet.

In the developed algorithm, this transition is modelled by setting the position equal to the limitation and recalculating the angular velocity and acceleration. This is implemented as follows. When the calculated position of a leaflet exceeds its limitations (i.e. $\theta_{i}^{n+1, k+1}<\theta_{\min }$ or $\theta_{i}^{n+1, k+1}>\theta_{\max }$ ), then the position is set equal to this limitation (i.e. $\theta_{i}^{n+1, k+1}=\theta_{\min }$ or $\left.\theta_{i}^{n+1, k+1}=\theta_{\max }\right)$. In order to move exactly to the limited position in the time step, the angular velocity $\dot{\theta}_{i}^{n+1, k+1}$ and acceleration $\ddot{\theta}_{i}^{n+1, k+1}$ are subsequently recalculated (using the backward Euler scheme):

$$
\begin{aligned}
& \theta_{i}^{n+1, k+1}=\theta_{\min } \text { or } \theta_{\max } \\
& \dot{\theta}_{i}^{n+1, k+1}=\frac{\theta_{i}^{n+1, k+1}-\theta_{i}^{n}}{\Delta t} \\
& \ddot{\theta}_{i}^{n+1, k+1}=\frac{\dot{\theta}_{i}^{n+1, k+1}-\dot{\theta}_{i}^{n}}{\Delta t}
\end{aligned}
$$

Furthermore, the previously described FSI convergence criterion must be changed when the position is limited since the FSI convergence criterion should no longer depend on the absolute value of $\left(M_{i}^{n+1, k}-I_{i} \cdot \ddot{\theta}_{i}^{n+1, k}\right)$, but merely on its mathematical sign. This can be understood by adding $M_{i}^{r, n+1, k}$ to the moment equation, with $M_{i}^{r, n+1, k}$ being the reaction moment of the blocking mechanism exerted on the leaflets in coupling iteration $k$ of time step $n+1$ :

$$
M_{i}^{r, n+1, k}+M_{i}^{n+1, k}=I_{i} \cdot \ddot{\theta}_{i}^{n+1, k}
$$

or

$$
M_{i}^{r, n+1, k}=-\left(M_{i}^{n+1, k}-I_{i} \cdot \ddot{\theta}_{i}^{n+1, k}\right)
$$

In order to maintain contact between the leaflet and the blocking mechanism, this reaction moment will be either negative or positive (depending on the leaflet and on the limiting position: $\theta_{\min }$ or $\theta_{\max }$ ). As FSI convergence criterion, it is checked whether or not the reaction moment has this appropriate mathematical sign. Therefore, when the motion of leaflet $i$ is limited, the FSI convergence criterion (Equation (12)) of this leaflet $i$ is replaced by

$$
M_{i}^{r, n+1, k} \leq 0
$$

or

$$
M_{i}^{r, n+1, k} \geq 0
$$

depending on the orientation of the leaflet and on whether the leaflet is fully open or fully closed.

Further details of the algorithm can be found in Annerel et al. (2011).

\subsection{Simulation setup}

The new quasi-Newton algorithm is used to simulate the dynamics of a 3D BMHV. This BMHV is a model of the $25 \mathrm{~mm}$ ATS Open Pivot ${ }^{\mathrm{TM}}$ Standard Heart Valve in aortic position with the orifice inner diameter measuring $20.8 \mathrm{~mm}$. The valve is simplified at the hinge regions by cutting away the blocking mechanism and hinges at the casing.
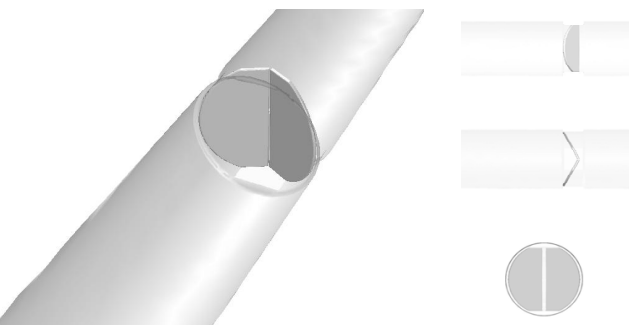

Figure 3: Views on the first geometry with straight tube downstream of the valve: isometric (left), from front side (up), from top side (middle) and from inlet (down).
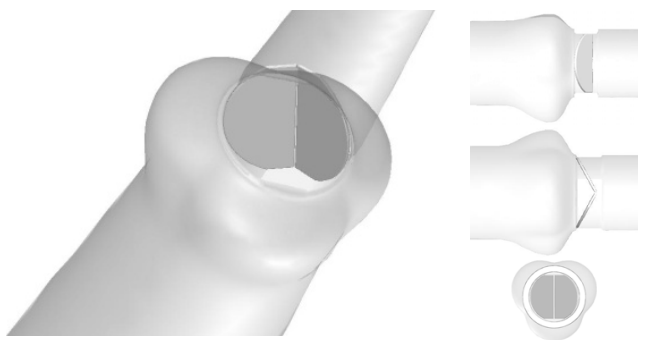

Figure 4: Views on the second geometry with symmetrically placed Valsalva sinuses downstream of the valve: isometric (left), from front side (up), from top side (middle) and from inlet (down).
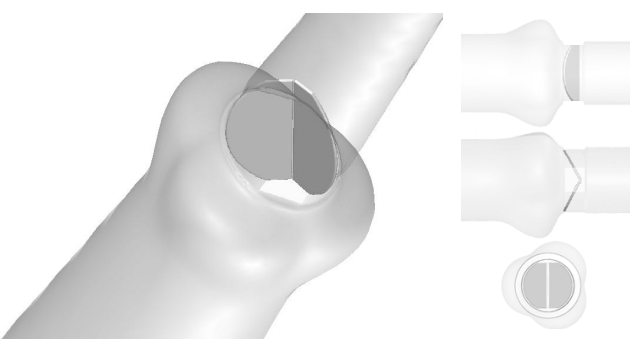

Figure 5: Views on the third geometry with asymmetrically placed Valsalva sinuses downstream of the valve: isometric (left), from front side (up), from top side (middle) and from inlet (down). 
The valve is subsequently placed in three geometries. The first geometry consists of a rigid straight tube (visualized in Figure 3). The second geometry also consists of a rigid straight tube upstream of the valve, but rigid Valsalva sinuses are added downstream of the valve. Such Valsalva sinuses are anatomically present in the ascending aortic root and have an influence on the valve closing. The Valsalva sinuses are based on the geometry described in Reul et al. (1990) and are positioned symmetrically with respect to the leaflets rotation axes (Figure 4). In the third geometry, the same Valsalva sinuses are used, but they are positioned asymmetrically (rotated over angle of 30 degrees) with respect to the leaflet rotation axes in such a way that one of the leaflets faces directly one sinus (Figure 5).

For the geometries, the upstream tube has a diameter of $22 \mathrm{~mm}$ and measures $75 \mathrm{~mm}$ in length. The downstream geometry has a length of $95 \mathrm{~mm}$. The diameter of the downstream tube is $27.36 \mathrm{~mm}$ for the Valsalva sinuses and $22 \mathrm{~mm}$ for the straight tube.

It is noted that these geometries are based on clincial practice since, when implanting the BMHV, the surgeon can choose to preserve the Valsalva sinuses or to cut them away and replace the entire ascending aortic root (in the so-called Bentall procedure (Bentall et al. 1968)). Moreover, the surgeon can choose to position the valve symmetrical to the anatomical Valsalva sinuses, or to position it asymmetrical. An unstructured grid is generated in the geometries, consisting of approximately 800000 tetrahedral cells. Two cell layers are generated in the gap (measuring $0.1 \mathrm{~mm}$ ) between the leaflets and the casing near the hinge region. The grids are constructed by defining the mesh size at the leaflet walls. Subsequently, a size function is applied at these leaflet walls, which means that in the direction perpendicular to the leaflet walls, the grid size in the geometry increases at a specified rate. Therefore, a very fine grid size can be obtained at the leaflet walls, resulting in an accurate calculation of the wall shear stress magnitude and distribution.

In previous study (Annerel et al. 2011), a spatial convergence study of the cases with the straight tube and the asymmetrical sinuses is performed. It is concluded that the described grid size is small enough to result in grid independent leaflet kinematics.

The ALE-approach is followed, which means that the fluid grid follows the motion of the structure and subsequently needs an update. This update is done using a local remeshing method and spring-based smoothing. Due to the ALE approach, this remeshing can only be performed when going to a new time step. During the subsequent coupling iterations, the grid motion is performed with spring-based smoothing in order to maintain good mesh quality. The maximum time step size $\Delta t^{\max }$ is set to $0.001 \mathrm{~s}$.

An inlet aortic flow pulse with a period of $1 \mathrm{~s}$ (displayed in Figure 6) is imposed upstream and was previously used in Dumont et al. (2005, 2007) and Annerel et al. (2010). The flow pulse profile is uniform. A physiological pressure profile is imposed at the downstream outlet boundary. Note, however, that in a rigid geometry the pressure level does not affect the flow field (only the pressure gradient appears in the equations).
Blood is modelled as an incompressible Newtonian fluid with density and viscosity respectively equal to $1050 \mathrm{~kg} / \mathrm{m}^{3}$ and $4 \mathrm{E}-3 \mathrm{~Pa} \cdot \mathrm{s}$. No turbulence model is used, thus implying laminar flow. A no-slip boundary condition is applied at the walls. The valve is initially set in the closed position. The moment of inertia of one rigid valve leaflet about its rotation axis is equal to $9.94 \mathrm{E}-9 \mathrm{~kg} \cdot \mathrm{m}^{2}$.

Each simulation is run in parallel on eight cores $(2 \mathrm{x}$ QuadCore Intel Xeon 2.66GHz) with 8GB RAM.

The FSI convergence threshold $\varepsilon_{0}$ is chosen as (with reference moment equal to $1 \mathrm{E}-9 \mathrm{Nm})$ :

$$
\varepsilon_{0}=\frac{M_{r e f}}{1 \mathrm{E} 4}=\frac{1 \mathrm{E}-9}{1 \mathrm{E} 4}=1 \mathrm{E}-13 \mathrm{Nm}
$$

The optimal values for $\varepsilon_{1}$ and $\varepsilon_{2}$ are derived from a sensitivity analysis and are equal to:

$$
\begin{aligned}
\varepsilon_{1} & =\frac{\varepsilon_{0}}{100} \\
\varepsilon_{2} & =0.001
\end{aligned}
$$

The algorithm is implemented in the commercial flow solver FLUENT ${ }^{\mathrm{TM}}$ (v.13.0.0, Ansys inc.). The NavierStokes equations are solved with second-order accuracy.

\section{$3 \quad$ Results}

In this section, the performance of the presented coupling algorithm is evaluated. Subsequently, the leaflet dynamics and the general flow field are discussed. Finally, the maximal wall shear stress that occurs during opening of the valve leaflets is studied in the three geometries.

\subsection{Solution process}

The average number of coupling iterations per time step for the first geometry is given in Table 1 . The solution process shows that during valve movement the developed algorithm reaches convergence in each time step within approximately four coupling iterations (see Table 1, "opening" and "closing", with $\left.\varepsilon_{0}=1 \mathrm{E}-13 \mathrm{Nm}\right)$.

Table 1: Averaged needed number of coupling iterations per time step for the presented coupling scheme, in the first geometry (straight tube) with $\varepsilon_{0}=M_{\text {ref }} / 10000, \varepsilon_{1}=\varepsilon_{0} / 100$ and $\varepsilon_{2}=0.001$.

\begin{tabular}{c|c|c|c|c}
$\begin{array}{c}\text { Opening } \\
(\mathrm{t}=0 \mathrm{~s} . .\end{array}$ & $\begin{array}{c}\text { Open } \\
(\mathrm{t}=0.0725 \mathrm{~s}\end{array}$ & $\begin{array}{c}\text { Closing } \\
(\mathrm{t}=0.325 \mathrm{~s} . .\end{array}$ & $\begin{array}{c}\text { Closed } \\
(\mathrm{t}=0.3925 \mathrm{~s}\end{array}$ & $\begin{array}{c}\text { Total } \\
(\mathrm{t}=0 \mathrm{~s} . .1 \mathrm{~s})\end{array}$ \\
\hline $0.0725 \mathrm{~s})$ & $. .0 .325 \mathrm{~s})$ & $0.3925 \mathrm{~s})$ & $. .1 \mathrm{~s})$ & \\
\hline 4.064 & 1 & 3.984 & 1 & 2.279
\end{tabular}

\subsection{Leaflet kinematics and flow field}

The results of the simulations are depicted in Figure 6 and Figure 7.

The angular positions of the leaflets are presented in Figure 6 , relative to the fully open position. Therefore, 0 and 1 refer, respectively, to the fully closed and fully open position. For the first geometry (straight tube), the leaflets of the valve reach the open position. Moreover, the resulting movement of the leaflets is symmetrical. Such symmetry in leaflet movement is also (approximately) found in the second geometry with symmetrical placed Valsalva sinuses. However, the leaflets do not reach the completely open position in this geometry. Finally, in the third geometry with asymmetrically placed Valsalva sinuses, both leaflets do not open completely and they do not behave symmetrically. 

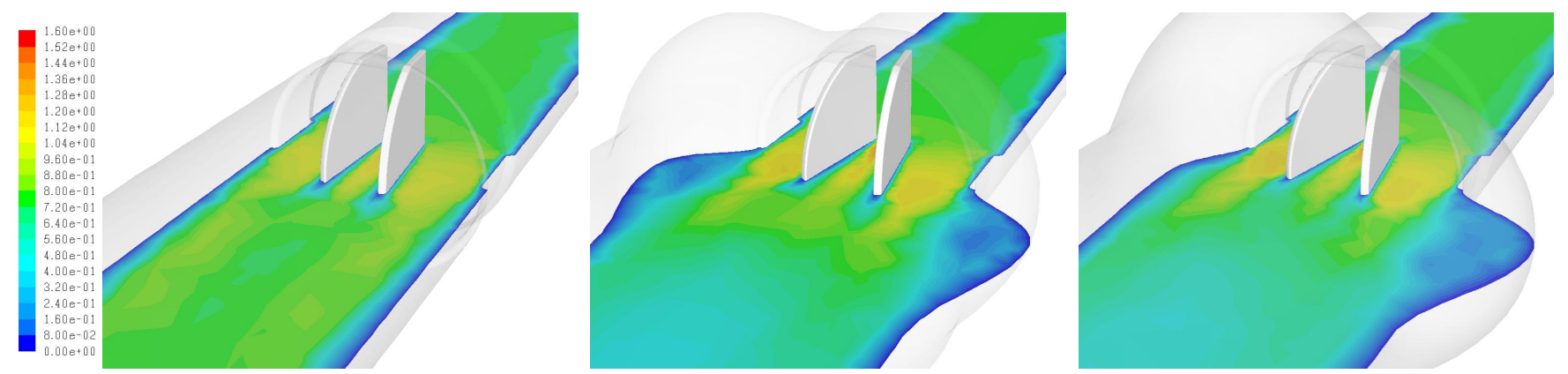

Figure 7: Velocity Magnitude in $\mathrm{m} / \mathrm{s}$ at peak systole $(t=0.125 \mathrm{~s})$ visualized on a longitudinal section perpendicular to the leaflet rotation axes, for the geometry with the straight tube (left), the symmetrically placed Valsalva sinuses (middle) and the asymmetrical Valsalva sinuses (right).

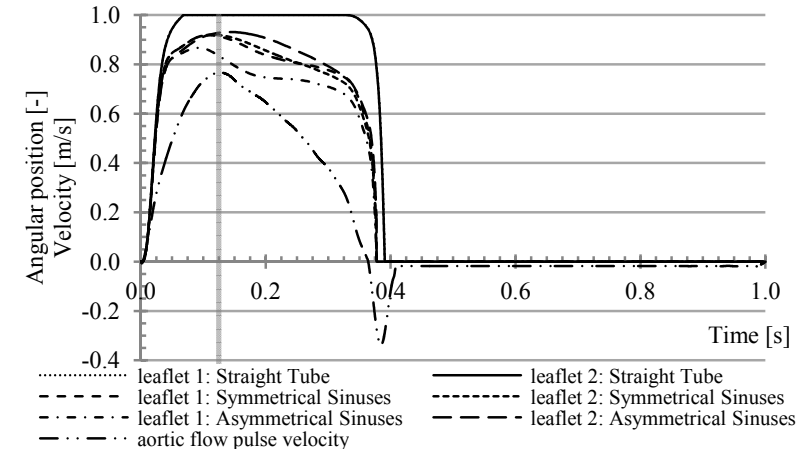

Figure 6: Angular position of the leaflets (relative to maximal opening angle) and the aortic flow pulse velocity. The leaflets of the straight tube geometry move symmetrically. The gray vertical line denotes "peak systole" $(t=0.125 \mathrm{~s})$, the time level at which Figure 7 is taken.

Figure 7 shows the velocity field for the geometries at peak systole. Downstream of the valve, the flow pattern consists of three jets, which is typical for a BMHV. The recirculation zones of the blood flow in each sinus of Valsalva is clearly visible. Furthermore, downstream these Valsalva sinuses, the flow velocity is lower than in the geometry with the straight tube due to the difference in downstream tube diameter.

The maximum Reynolds number during forward flow is approximately 4400 .

\subsection{Maximal wall shear stress on the valve leaflets}

The amount of blood damage can be related to the magnitude and the duration (exposure time) of the applied shear stresses (Dumont et al. 2007). In this section, the magnitudes of the wall shear stresses on the valve leaflets are discussed. Since the difference in leaflet opening movement between the three geometries is a main interest of this paper, only the forward flow is studied.

In all geometries, the maximal wall shear stress during forward flow is observed at $t=0.125 \mathrm{~s}$ (peak systole). Furthermore, because this time level corresponds with the maximal blood inflow velocity, the maximal wall shear stress is located at the (b-datum) leading edge of the leaflets, as is visualized in Figure 8. For the first geometry (with straight tube), a maximal wall shear stress of $197.8 \mathrm{~Pa}$ is calculated at both leaflets. In the second geometry (with symmetrically placed Valsalva sinuses), the magnitude of the maximal wall shear stress is approximately the same: 192.5Pa for both leaflets. Finally, the third geometry (with asymmetrically placed Valsalva sinuses), induces the same value of $197.2 \mathrm{~Pa}$ at one leaflet, but a significantly larger maximal wall shear stress of $233.3 \mathrm{~Pa}$ is observed at the other leaflet (i.e. at "leaflet 2", the leaflet that faces directly one sinus).

Subsequently, the wall shear stresses are spatially averaged on the leaflets during peak systole. This results for both leaflets in an average wall shear stress of $16.5 \mathrm{~Pa}$ in the first geometry, and an average wall shear stress of $17.2 \mathrm{~Pa}$ in the second geometry. For the third geometry, however, a slightly larger value of $17.9 \mathrm{~Pa}$ is calculated for "leaflet 2 " and a much larger average shear stress of $18.2 \mathrm{~Pa}$ is observed on the other leaflet. The values of the average shear stress are in the same magnitude as calculated in previous studies (Dumont et al. 2005, 2007).
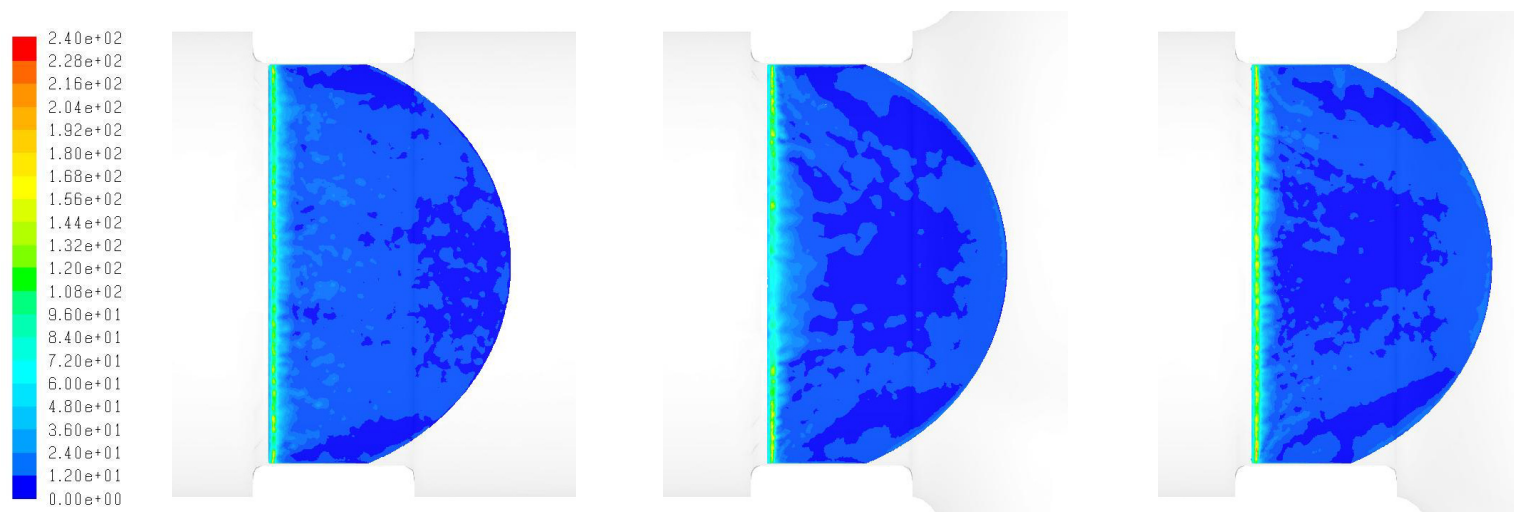

Figure 8: Distribution of wall shear stress magnitude in Pa on one valve leaflet surface during peak systole $(t=0.125 \mathrm{~s})$, front view seen from the b-datum gap, for the geometry with the straight tube (left), the symmetrically placed Valsalva sinuses (middle) and leaflet 2 of asymmetrical Valsalva sinuses (right). 


\section{Discussion}

The results indicate that the presented algorithm has a stable and efficient solution process.

Furthermore, the resulting leaflet positions agree well with Feng et al. (1999, 2000) and De Tullio et al. (2009).

In particular, the results show that the maximum open position of the valve leaflets is not reached in the geometries with the Valsalva sinuses. Such incomplete opening for the ATS Open Pivot ${ }^{\mathrm{TM}}$ Standard Heart Valve in a divergent geometry is explained due to the greater sensitivity of the leaflet movement to the downstream flow field compared to other BMHV designs, since the leaflets extend farther in the flow downstream of the orifice than is the case in other designs. Therefore, the valve does not open completely in the divergent transvalvular flow caused by the Valsalva sinuses enlargement, since the leaflets tend to align with the streamlines (Feng et al. 1999, 2000). In the straight tube, however, a full opening of the valve leaflets is observed.

Moreover, it is observed that the two leaflets have differences in movement for the third geometry (with asymmetrically placed Valsalva sinuses). This asynchrony is triggered by the presence of the asymmetry in the geometry downstream of the valve. In the symmetrical geometries, no differences in movement between the two leaflets are observed. The asynchronous leaflet movement caused by the asymmetrically placed Valsalva sinuses is consistent with De Tullio et al. (2009).

Finally, for forward flow, the maximal wall shear stresses generated at a valve leaflet is significantly lower in the symmetrical geometries than is the case in the geometry with asymmetrically placed Valsalva sinuses. Because the maximal magnitude of wall shear stress is related to the acquired blood damage, it can be concluded that, in case of the ATS Open Pivot ${ }^{\mathrm{TM}}$ Standard Heart Valve, placing the downstream geometry symmetrically with respect to the valve leaflets reduces the accumulated amount of blood damage during forward flow.

\section{Conclusion}

In this paper, a strong coupling scheme for the partitioned simulation of a BMHV is presented and evaluated. The convergence of the coupling iterations between the flow solver and the leaflet motion solver is accelerated using the Jacobian with the derivatives of the pressure and viscous moments acting on the leaflets with respect to the leaflet accelerations. This Jacobian is numerically calculated from the data in the coupling iterations. A criterion is used for the selection of useable coupling iterations.

Subsequently, the algorithm is used to simulate an ATS Open Pivot ${ }^{\mathrm{TM}}$ Standard Heart Valve in three different geometries. The results show that the presented scheme is stable and converges within a few coupling iterations. Moreover, it is observed that the leaflets tend to align with the streamlines. As a consequence, the leaflets perform an asynchronous movement in an asymmetric (downstream) geometry and they do not open completely in a divergent downstream geometry. Since symmetrical downstream geometries induce the smallest wall shear stress during systole, such geometries are preferred for valve replacement.

\section{Acknowledgements}

Sebastiaan Annerel is funded by a BOF-grant (Special Research Fund) from Ghent University Association. Joris Degroote gratefully acknowledges a postdoctoral fellowship of the Research Foundation - Flanders (FWO).

The paper is originally presented at $8^{\text {th }}$ International conference on CFD in oil and gas, metallurgical and process industries (2011), Trondheim, Norway. However, the accuracy of the simulations described in the conference paper is only first order. Therefore, in this paper, the results are improved by performing the simulations with a second order accuracy.

\section{References}

Annerel, S., Degroote, J., Claessens, T., Vierendeels, J., (2010), Evaluation of a new Implicit Coupling Algorithm for the Partitioned Fluid-Structure Interaction Simulation of Bileaflet Mechanical Heart Valves, IOP Conf Ser: Mater Sci Eng, 10, 012124.

Annerel, S., Degroote, J., Claessens, T., Dahl, S.K., Skallerud, B., Hellevik, L.R., Van Ransbeeck, P., Segers, P., Verdonck, P., Vierendeels, J., (2011), A Fast Strong Coupling Algorithm for the Partitioned Fluid-Structure Interaction Simulation of BMHVs. Comput Method Biomec. In press.

Bang, J.S., Yoo, S.M., Kim, C.N., (2006), Characteristics of Pulsatile Blood Flow Through the Curved Bileaflet Mechanical Heart Valve Installed in Two Different Types of Blood Vessels: Velocity and Pressure of Blood Flow, ASAIO J, 52, 234-242.

Bentall, H., De Bono, A., (1968), A technique for complete replacement of the ascending aorta, Thorax, 23, 338-339.

Choi, C.R., Kim, C.N., (2009), Numerical Analysis on the Hemodynamics and Leaflet dynamics in a Bileaflet Mechanical Heart Valve Using a Fluid-structure Interaction Method, ASAIO J., 55(5), 428-437.

Dahl, S.K., Vierendeels, J., Degroote, J., Annerel, S., Hellevik, L.R., Skallerud, B., (2010), FSI-simulation of asymmetric mitral valve dynamics during diastolic filling, Comput Method Biomec, DOI: 10.1080/10255842.2010.517200.

Dasi, L.P., Simon, H.A., Sucosky, P. \& Yoganathan, A.P., (2009), Fluid mechanics of artificial heart valves, Clin Exp Pharmacol Physiol, 36, 225-237.

De Tullio, M.D., Cristallo, A., Balaras, E., Verzicco, R., (2009), Direct numerical simulation of the pulsatile flow through an aortic bileaflet mechanical heart valve, J Fluid Mech, 622, 259-290.

Dumont, K., Vierendeels, J., Segers, P., Van Nooten, G., Verdonck, P., (2005), Predicting ATS Open Pivot ${ }^{\mathrm{TM}}$ Heart Valve Performance with Computational Fluid Dynamics, $J$ Heart Valve Dis, 14, 393-399.

Dumont, K., Vierendeels, J., Kaminsky, R., Van Nooten, G., Verdonck, P., Bluestein, D., (2007), Comparison of the Hemodynamic and Thrombogenic Performance of Two 
Bileaflet Mechanical Heart Valves Using a CFD/FSI Model, J Biomech Eng, 129, 558-565.

Feng, Z., Umezu, M., Fujimoto, T., Tsukahara, T., Nurishi, M., Kawaguchi, D., Masuda, S., (1999), Analysis of ATS leaflet behaviour by in vitro experiment, J Artif Organs, 2, 46-52.

Feng, Z., Umezu, M., Fujimoto, T., Tsukahara, T., Nurishi, M., Kawaguchi, D. (2000). In Vitro Hydrodynamic Characteristics Among Three Bileaflet Valves in the Mitral Position, J Artif Organs, 24(5), 346-352.

Guivier, C., Deplano, V., Pibarot, P., (2007), New insights into the assessment of the prosthetic valve performance in the presence of subaortic stenosis through a fluid-structure interaction model, J Biomech, 40, 2283-2290.

Reul, H., Vahlbruch, A., Giersiepen, M., Schmitz-Rode, T.h., Hirtz, V., Effert, S., (1990), The geometry of the aortic root in health, at valve disease and after valve replacement, J Biomech, 23, 181-191.

Vierendeels, J., Dumont, K., Dick, E., Verdonck, P., (2005), Analysis and Stabilization of Fluid-Structure Interaction Algorithm for Rigid-Body Motion, AIAA J, 43, 2549-2557.

\section{Nomenclature}

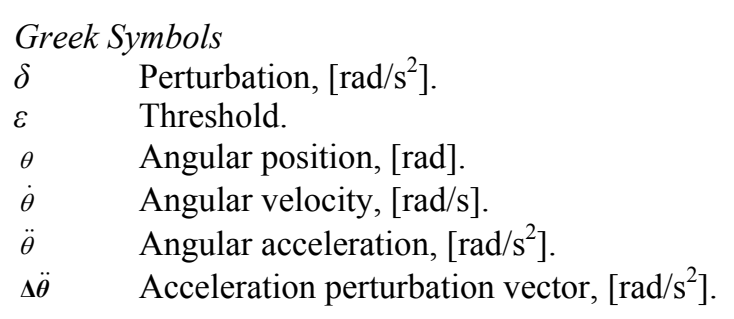

Latin Symbols

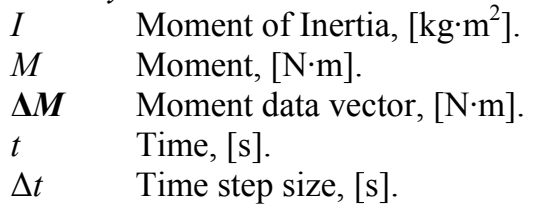

\section{Subscripts}

$0 \quad$ FSI convergence threshold.

$1 \quad$ First leaflet;

Flow solver convergence threshold.

2 Second leaflet;

Jacobian update threshold.

$i, j \quad$ Leaflet.

max Maximal value.

min Minimal value.

ref Reference.

\section{Superscripts}

$\begin{array}{ll}a & \text { First acceleration perturbation vector. } \\ b & \text { Second acceleration perturbation vector. } \\ k & \text { Coupling iteration. } \\ n & \text { Time step. } \\ r & \text { Reaction moment. } \\ r e f & \text { Reference. }\end{array}$

